\title{
Interplay of intrinsic and network heterogeneity in strongly recurrent spiking networks
}

\author{
Cheng Ly \\ From 24th Annual Computational Neuroscience Meeting: CNS*2015 \\ Prague, Czech Republic. 18-23 July 2015
}

Heterogeneity has recently gained a lot of attention and it is becoming more apparent that it is a crucial feature in neural processing [1-5]. Despite its importance, this realistic physiological feature has traditionally been neglected in theoretical studies of cortical neural networks. A common reason is that mean-field descriptions of noisy cortical networks are high dimensional and generally intractable. Although heterogeneous spiking neural networks have recently been studied theoretically [5-8], there is still a lot unknown. In particular, combining network heterogeneity [9] and intrinsic heterogeneity [10] have yet to be considered simultaneously despite the fact that both are known to exist and likely have significant roles in neural network dynamics.

To this end, we study a recurrently coupled spiking network of leaky integrate-and-fire (LIF) neurons consisting of excitatory and inhibitory neurons. The intrinsic heterogeneity is modeled by varying the voltage threshold for spiking [5], and the network heterogeneity is modeled by different conductance strengths (partially motivated by recent results [11], both excitatory and inhibitory conductances are scaled so each neuron has a different level of balanced input). Unsurprisingly, we find that when either intrinsic or network heterogeneity is increased, the response heterogeneity also increases (i.e., the range of the average firing rate of the excitatory neurons also increases). However, for a fixed level of both forms of heterogeneity, the network robustly exhibits a wide range of response heterogeneity that strongly depends on the relationship between intrinsic and network heterogeneity. This coupled network is difficult to analyze because it is stochastic, heterogeneous, and high dimensional with alpha function synapses and colored external noisy input. With combination of Monte Carlo simulations and

Correspondence: CLy@vcu.edu

Department of Statistical Sciences and Operations Research, Virginia Commonwealth University, Richmond, VA 23284, USA augmented mean-field theory based partially on methods in [12-15], we provide analytic explanations to account for the observed phenomena. Our work gives insight for how these two forms of heterogeneity interact in a generic recurrent spiking network that may be applicable to many areas of the cortex.

Published: 18 December 2015

\section{References}

1. Shamir M, Sompolinsky H: Implications of neuronal diversity on population coding. Neural Computation 2006, 18:1951-1986.

2. Chelaru MI, Dragoi V: Efficient coding in heterogeneous neuronal populations. Proceedings of the National Academy of Sciences 2008, 105:16344-16349.

3. Padmanabhan K, Urban NN: Intrinsic biophysical diversity decorrelates neuronal firing while increasing information content. Nature neuroscience 2010, 13:1276-1282.

4. Tripathy SJ, Padmanabhan K, Gerkin RC, Urban NN: Intermediate intrinsic diversity enhances neural population coding. Proceedings of the National Academy of Sciences 2013, 110:8248-8253.

5. Mejias JF, Longtin A: Optimal heterogeneity for coding in spiking neural networks. Physical Review Letters 2012, 108:228102.

6. Ly C: Dynamics of Coupled Noisy Neural Oscillators with Heterogeneous Phase Resetting Curves. SIAM Journal on Applied Dynamical Systems 2014, 14:1733-1755.

7. Nicola W, Campbell SA: Mean-field models for heterogeneous networks of two-dimensional integrate and fire neurons. Frontiers in computational neuroscience 2013, 7:184.

8. Zhou P, Burton SD, Urban NN, Ermentrout GB: Impact of neuronal heterogeneity on correlated colored noise-induced synchronization. Frontiers in computational neuroscience 2013, 7:113.

9. Perin R, Berger TK, Markram H: A synaptic organizing principle for cortical neuronal groups. Proceedings of the National Academy of Sciences 2011, 108:5419-5424.

10. Marder E: Variability, compensation, and modulation in neurons and circuits. Proceedings of the National Academy of Sciences 2011, 108:15542-15548.

11. Xue M, Atallah BV, Scanziani M: Equalizing excitation-inhibition ratios across visual cortical neurons. Nature 2014, 511:596-600.

12. Moreno-Bote R, Parga N: Auto- and crosscorrelograms for the spike response of leaky integrate-and-fire neurons with slow synapses. Physical Review Letters 2006, 96:028101.

13. Nesse WH, Borisyuk A, Bressloff PC: Fluctuation-driven rhythmogenesis in an excitatory neuronal network with slow adaptation. Journal of Computational Neuroscience 2008, 25:317-333. 
14. Ly C: A Principled Dimension-Reduction Method for the Population Density Approach to Modeling Networks of Neurons with Synaptic Dynamics. Neural Computation 2013, 25:2682-2708.

15. Nicola W, Ly C, Campbell SA: One-dimensional Population Density Approaches to Recurrently Coupled Networks of Neurons with Noise [http:// arxiv.org/abs/1411.2273], Submitted.

doi:10.1186/1471-2202-16-S1-P150

Cite this article as: Ly: Interplay of intrinsic and network heterogeneity

in strongly recurrent spiking networks. BMC Neuroscience 2015

16(Suppl 1):P150.

Submit your next manuscript to BioMed Central and take full advantage of:

- Convenient online submission

- Thorough peer review

- No space constraints or color figure charges

- Immediate publication on acceptance

- Inclusion in PubMed, CAS, Scopus and Google Scholar

- Research which is freely available for redistribution

Submit your manuscript at www.biomedcentral.com/submit

() BioMed Central 